\title{
Interactions entre recherches fondamentale et clinique. Deux exemples tirés d'une expérience personnelle
}

\author{
D. Albe-Fessard \\ Laboratoire de physiologie de la nutrition, \\ Institut national de recherche agronomique, France
}

\begin{abstract}
RÉSUMÉ: Les recherches cliniques bénéficient des apports de la recherche fondamentale, et inspirent celle-ci. Deux études en servent d'exemples: celles conduites pour comprendre l'origine des anomalies que présente l'encéphale chez les malades parkinsoniens, et les patients atteints d'un syndrome douloureux produit par une désafférentation. L'évolution au cours des 40 dernières années, des recherches inspirées par ces deux problèmes (et en particulier de celles réalisées par l'équipe dont faisait partie l'auteur), est replacée dans le contexte international. Le précision de la stéreotaxie chez l'Homme a été améliorée grâce aux connaissances réunies par la physiologie animale sur l'organisation de la projection thalamique des afférences somatiques. La présence dans le thalamus des malades parkinsoniens, de cellules pulsant au rythme du tremblement, a conduit à l'étude des relations qui existent entre le système striato-nigral et le thalamus moteur. Chez les patients ressentant des douleurs après privation d'afférences, un rôle particulier semble joué par la région du thalamus médian dont les propriétés avaient jusqu'ici été étudiées chez l'animal. L'origine de ces douleurs est actuellement recherchée sur un modèle animal.
\end{abstract}

\begin{abstract}
Interaction between basic and clinical research: Two examples from personal experience Basic research helps to understand clinical knowledge which in turn leads to new fundamental research. Two examples are taken from the studies of the anomalies existing in the brain of patients suffering from Parkinson disease or deafferentation Pain. The researches performed to solve these two problems for the past 40 years (in particular by the author's working group) are replaced in the international context. In Man the precision of stereotaxic technique was improved by using the organisation of the somato-sensory thalamic projection described in animals by the physiologists. In the thalamus of Parkinsonian patients, the presence of cells bursting at tremor frequency led to search for the relations existing between the striato-nigral system and the motor thalamus. In the patients suffering from deafferentation Pain, a special role is attributed to the medial thalamus, whose characteristics were formerly studied in animals. The origin of the deafferentation Pain is nowadays studied in an animal model of this disease.
\end{abstract}

Can. J. Neurol. Sci. 1988; 15:324-332

Les lésions créées par la Maladie au niveau du système nerveux humain, constituent des conditions expérimentales spontanées qui ont bien souvent inspiré des recherches fondamentales. Deux exemples serviront à illustrer cette affirmation, mais auparavant afin d'expliquer comment nous avons été conduits à réaliser ces recherches, je résumerai mes débuts dans la carrière scientifique.

Ma formation n'est pas médicale. J'ai appris dans une école d'ingénieur, parmi d'autres techniques, à construire des amplificateurs, ceci à une époque où l'industrie ne fournissait pas encore ce matériel aux Physiologistes. Cette spécialisation m'a conduite à travailler avec des neurophysiologistes, et à m'intéresser à l'enregistrement des signaux nerveux. J'ai tout d'abord tenté de comprendre comment les poissons électriques qui ne disposent que des éléments excitables voisins de ceux existant chez les mammifères, pouvaient émettre des trains d'impulsions de voltage élevé (120 à 180 volts en circuit fermé chez le gymnote), prouesse que les mammifères sont incapables d'accomplir. Pour le faire, ils doivent activer presque simultanément des piles élémentaires de 0.1 volts, placées en série par une disposition anatomique. Au cours de ces recherches réalisées avec A. Fessard, C. Chagas et H. Martins-Fereira nous avons démontré, chez le gymnote, que la simultanéité de ces décharges se produisait grâce à un centre rythmeur bulbaire: ce dernier envoie des ordres qui, afin de compenser les délais de conduction, sont différemment retardés au niveau des synapses médullaires. Ainsi ce premier travail m'avait amenée à étudier le fonctionnement de structures du système nerveux central, et conduite à employer la microphysiologie. Celle-ci venait en effet d'être développée dans la moelle, au niveau intracellulaire, 
par J. Eccles et ses collaborateurs. ${ }^{1}$ A la suite de Ladislav Tauc nous l'avons utilisée au laboratoire. Après avoir construit les changeurs d'impédance indispensables, chacun de nous a pu l'appliquer au problème qu'il étudiait alors. Des dérivations intracellulaires dans les électroplaques puis dans les cellules des centres de commande bulbaire chez les poissons électriques ont ainsi été réalisées. Rapidement aussi ces techniques, appliquées chez le mammifère au niveau des cellules du cortex somatomoteur, ont montré que des hyperpolarisations de longue durée accompagnent la phase négative des potentiels évoqués. ${ }^{2}$

L'exploration des structures profondes par la méthode stéréotaxique, associée à la dérivation par électrodes bipolaires concentriques, était employée chez le chat, depuis plusieurs années par H. Magoun. Nous avons appliqué stéréotaxie, macro- et microdérivations afin d'étudier les activités du thalamus non-spécifique. A l'époque où débutaient nos observations chez le mammifère un autre groupe étudiait déjà, avec des méthodes voisines, les réponses observées dans le thalamus spécifique. A la suite de Mountcastle et Henneman ${ }^{3,4}$ qui utilisaient des macroélectrodes, Rose et Mountcastle ${ }^{5}$ avaient mis en oeuvre à ce niveau la microphysiologie et démontré que, chez le chat, des réponses contralatérales apparaissent au niveau du noyau ventral postérieur du thalamus. La somatotopie qu'ils observaient dans cette structure rappelait celle déjà décrite au niveau du cortex (Adrian, 6 ; Marshall et al; 7 Woolsey et Fairman, ${ }^{8}$ ). Tentant de reproduire ces données classiques, nous nous aperçumes qu'il existait, dans des zones bien localisées du cortex des réponses qui n'avaient pas cette organisation somatotopique. Ces aires recevaient leurs afférences à partir de toutes les régions du corps aussi bien ipsi- que contralatérales (l'une d'entr'elles avait déjà été décrite par Amassian, ${ }^{9}$ ). Recherchant le relai thalamique placé sur la voie de ces afférences convergentes, nous étudiâmes par la stéréotaxie les réponses du noyau dorso-médian, qui était considéré à l'époque, comme la structure associative de la somesthésie. A notre surprise ce n'est pas dans ce noyau, mais dans une région immédiatement inférieure, appelée centre médian dans l'atlas que venaient de publier Jasper et Ajmone Marsan, ${ }^{10}$ que se trouvaient les réponses ayant des caractéristiques qui rappelaient celles que nous avions trouvées au niveau du cortex. Ces réponses convergentes occupaient chez le chat, toute la région comprenant le noyau parafasciculaire, le centre médian (CM) et le noyau centralis lateralis." Nous appelerons ici, par simplification, thalamus médian cette région el ne discuterons pas de l'existence chez les carnivores du noyau centre médian (voir pour ce problème) ${ }^{12}$ Les réponses observées dans le thalamus médian avaient des caractéristiques bien différentes de celles observées dans le noyau ventral postérieur voisin. Jersey Rose, après avoir pensé que nous commettions une erreur de structure, fut persuadé lorsque son élève, Laurence Kruger, qui était venu travailler avec nous, confirma la localisation anatomique de la région thalamique qui présentait les réponses convergentes.

Nos résultats continuaient cependant d'être mis en doute par V. Mountcastle. L'explication était simple: le groupe américain utilisait comme anesthésique des barbituriques alors que nous employions le chloralose. L'étape suivante a donc été pour nous de prouver que les réponses du CM existaient bien chez l'animal non anesthésié (résultat qui avait d'ailleurs été déjà obtenu par Magoun et Mac Kinley). ${ }^{13}$ C'est à partir de cette époque que nous avons dans la plupart de nos expériences renoncé à utiliser l'anesthésie au chloralose et travaillé chez des animaux en préparation chronique ou n'ayant subi qu'une anesthésie légère puis curarisés et placés sous respiration artificielle, dans des conditions inspirées de celles employées en clinique humaine.

\section{Recherches sur le syndrome Parkinsonien}

Grâce aux recherches que nous venons de résumer nous étions entrés en contact avec les anesthésistes et les ncurochirurgiens. Au cours d'une conférence organiséc par le Professeur Le Beau, le neurochirurgien G. Guiot souligna le manque de précision que présentait la stéréotaxie chez l'homme. A cette époque, la neurochirurgie fonctionnelle était très utilisée, il n'existait pas en effet d'autre moyen pour soigner les Parkinsoniens. Des lésions étaient pratiquées chez ces patients dans le noyau ventral latéral ou le noyau qui lui est juste supérieur c'est à dire dans des régions avoisinant le n. Ventral Postérieur (VP) et la capsule motrice. Pour cette raison, les opérations se soldaient souvent par des déficits moteurs ou des paresthésies lorsque la lésion, placée à partir de données anatomiques moyennes et des repères radiologiques intracérébraux, avait débordé sur la capsule ou sur le n.VP. Les neurochirurgiens, à cette époque, se protégeaient contre ces erreurs en employant des stimulations intracérébrales. Celles-ci lorsqu'elles sont constituées de choc isolés el appliquées à la capsule provoquent des mouvements de flexion. Organisées en trains d'impulsions elles produisent mouvement où sensations lorsque elles sont appliquées dans le thalamus somatique. Malgré ces précautions, la localisation des régions qui supprimaient efficacement les tremblements dans le bras et la jambe. demeurait souvent imprécise. J'ai suggéré de corriger les erreurs de localisation en employant une méthode qui s'avérait fournir, chez le chat, des repères indiscutables, l'enregistrement dans le n.VP des activités évoquées par la stimulation naturelle des différentes parties du corps, celles-ci devaient avoir chez I'homme l'organisation topique trouvée chez l'animal. Avec Gérard Guiot, nous avons rapidement commencé à utiliser l'électrophysiologie pour corriger les coordonnées stéréotixiques déterminées par la radiologie. Nous formions à celle époque une équipe soudée qui comprenait outre moi-même ct un technicien, le neurochirurgien mais aussi le neuro-radiologue (E. Hertzog), l'anesthésiste (G. Vourc'h), l'electroencéphalographiste (G. Arfel), le neurologue (S. Brion) et un neurochirurgien canadien (Jules Hardy). Nous utilisions les électrodes bipolaires concentriques que nous employions déjà chez. l'animal, notre technicien les ayant seulement construites avec des tubes plus longs afin qu'elles puissent passer au travers du tube guide, à la place que prendrait ensuite l'électrode de coagulation. Au cours de ces premières expériences, le matériel électrophysiologique était transporté plusieurs jours par semaine de mon laboratoire expérimental jusqu'à l'hôpital. Les résultats obtenus justifiaient cet effort. La stéréotaxie, telle que l'employait G. Guiot, comprenait une approche postérieure, si bien qu'il était aisé de rencontrer le n.VP sur la trajectoire qui se dirigeait vers une région juste antérieure à ce noyau. Nous avions donc. par les limites de la zone qui contenait des activités évoquées de type primaire, une bonne appréciation des coordonnées de la région thalamique qu'il convenait de détruire. En outre, après avoir réalisé un certain nombre de trajectoires, nous avons pu confirmer que la somatotopie, classique chez le chat, pouvait 
être observée chez l'homme: la représentation du pied et de la jambe se trouvait placée près de la capsule interne, la représentation du bras et de la main, un peu plus médialement, la représentation de la face se trouvant encore plus médiale et celle de la langue sous le noyau centre médian. Les corrections des coordonnées latérales ont donc pu être effectuées en tenant compte de la zone du corps dont la représentation avait été trouvée au cours d'une trajectoire. ${ }^{14,15}$

Les améliorations techniques n'allèrent pas uniquement du laboratoire vers la clinique; nos méthodes expérimentales y gagnèrent aussi en précision. La stéréotaxie classiquement employée chez le Singe était alors celle développée par Horsley et Clarke, ${ }^{16}$ elle était basée comme celle utilisée chez le chat, sur l'existence de points de repères osseux. Or, chez tous les primates, cette méthode est inexacte. Les centres encéphaliques

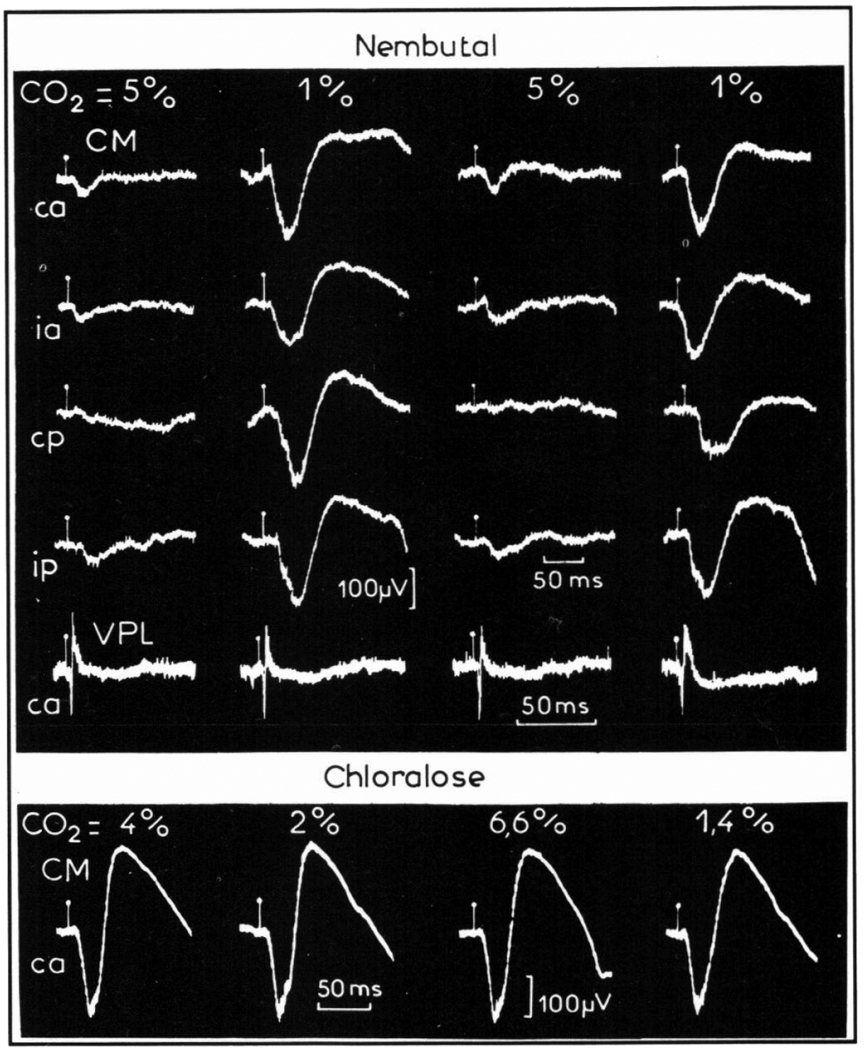

Figure I - Activités évoquées dans le thalamus médian (CM) et le $n$. ventral postérieur latéral (VPL) chez deux chats, l'un soumis à une anesthésie légère au nembutal (tracés du haut) l'autre à une anesthésie au chloralose (tracés du bas). puis curarisés et placés sous respiration arrificielle. Stimulation électrique des extrémités des membres antérieurs contra (ca)- et ipsilatéral (ia) et postérieurs contra (cp)-et ipsilatéral (ip). Dans le cas du VPL les effets provoqués par la stimulation du membre activant la zone enregistrée (ca) sont seuls présentés. La mesure par un capnographe de la concentration de CO2 au niveau de la trachée est porté en haut de la figure (CO2), des modifications de celle-ci ont été provoquées en modifiant le volume d'air inspiré (1 à $6,6 \%)$.

Tracés du haut-Les réponses n'apparaissent au niveau du CM que pour un miveau faible de CO2. elles-ci sont dans ce cas produites par la stimulation des 4 membres. Les réponses du n.VP sont inchangées.

Tracés du bas-Expérience similaire pratiquée sous chloralose. Aucune modification des réponses n'est observée dans le CM dont seule une réponse est présentée ici (d'après $2 I$ ). n'ont pas une position constante dans le crâne chez les individus d'une même espèce, de poids égal et de même sexe, et a fortiori les variations deviennent très importantes quand poids et sexe dont différents. ${ }^{17,18}$ Comme l'ont montré Talairach et al 18 pour l'homme, des repères intracérébraux choisis sur les radiographies des ventricules permettent une stéréotaxie plus précise. Nous avons appliqué ce principe à la stéréotaxie du macaque et amélioré ainsi considérablement les résultats que nous obtenions chez l'animal. 20 Il est aisé à partir d'une radiographie des ventricules injectés d'un liquide de contraste, de déterminer les coordonnées stéréotaxiques de la commissure postérieure (CP) et de la commissure antérieure (CA), et de corriger ainsi les coordonnées des structures d'un animal donné en utilisant un atlas classique et en pratiquant, s'il s'agit d'un sujet adulte, une simple translation. Pour les très jeunes animaux, les corrections sont plus complexes et nécessitent homothétie et rotation. Nous avons appris aussi à l'époque à mieux manier l'anesthésie et à employer pour la contrôler la mesure du niveau de gaz carbonique ( $\mathrm{CO2}$ ) alvéolaire grâce au capnographe que certains anesthésistes employaient alors déjà en salle d'opération. Ce type d'observation nous a permis de mieux comprendre les effets opposés qu'ont, sur les réponses évoquées du thalamus médian, les anesthésies aux barbituriques et au chloralose. Les animaux sous anesthésie barbiturique sont souvent hypercapniques et il suffit de les rendre hypocapniques pour faire apparaître les réponses lentes du CM, (figure 1). Nous avons aussi, en collaboration avec Wade Marshall, pu montrer que l'activité des cellules était fortement affectée par le niveau de $\mathrm{CO} 2$ alvéolaire et que l'activité normale des unités ne pouvait être observée chez l'animal anesthésié que dans une marge étroite, celle de la normocapnie de l'animal éveillé.

Les électrodes bipolaires, que nous employions, permettaient d'observer les ondes lentes évoquées mais aussi, lorsqu'elles étaient convenablement affutées, de recueillir les activités spontanées et provoquées d'unités cellulaires. C'est au cours d'une exploration réalisée pour rechercher la zone thalamique antérieure et supérieure par rapport au n.VP, région où G. Guiot pratiquait alors les lésions curatives chez les Parkinsoniens, que nous observâmes pour la première fois chez l'homme, des cellules ayant une activité en bouffées se succédant à la fréquence du tremblement. ${ }^{15,22}$ Par la suite, des activités similaires ont rapidement été trouvées par nous-même puis par d'autres au Canada, au Japon et en Russie, dans une zone qui entoure vers le haut et l'avant le noyau Ventral Postérieur et qui est constituée du Ventral Intermediaire (Vim) de la nomenclature employée par les anatomistes allemands, d'une partie du n.Latéral Postérieur ainsi que de la zona incerta (figure 2). C'est dans le Vim que vont se projeter les afférences provenant des muscles ainsi que nous avons pu le montrer, chez le macaque, avec Yves Lamarre. De plus, cette région transmet ses afférences vers le cortex moteur, où des réponses évoquées ont été chez le singe décrites par Woolsey et al ${ }^{25}$ et dont les cellules reçoivent les afférences provoquées par les mouvements des membres et les récepteurs intramusculaires, ainsi que nous l'avons montré avec J. Liebeskind. ${ }^{23}$ Une disposition analoque existe aussi, selon toute probabilité, chez l'homme. Ces données ont tout d'abord été contestées en raison du dogme selon lequel les influx provenant du thalamus primaire ne se projettent que sur la pariétale ascendante, cette projection étant limitée vers 


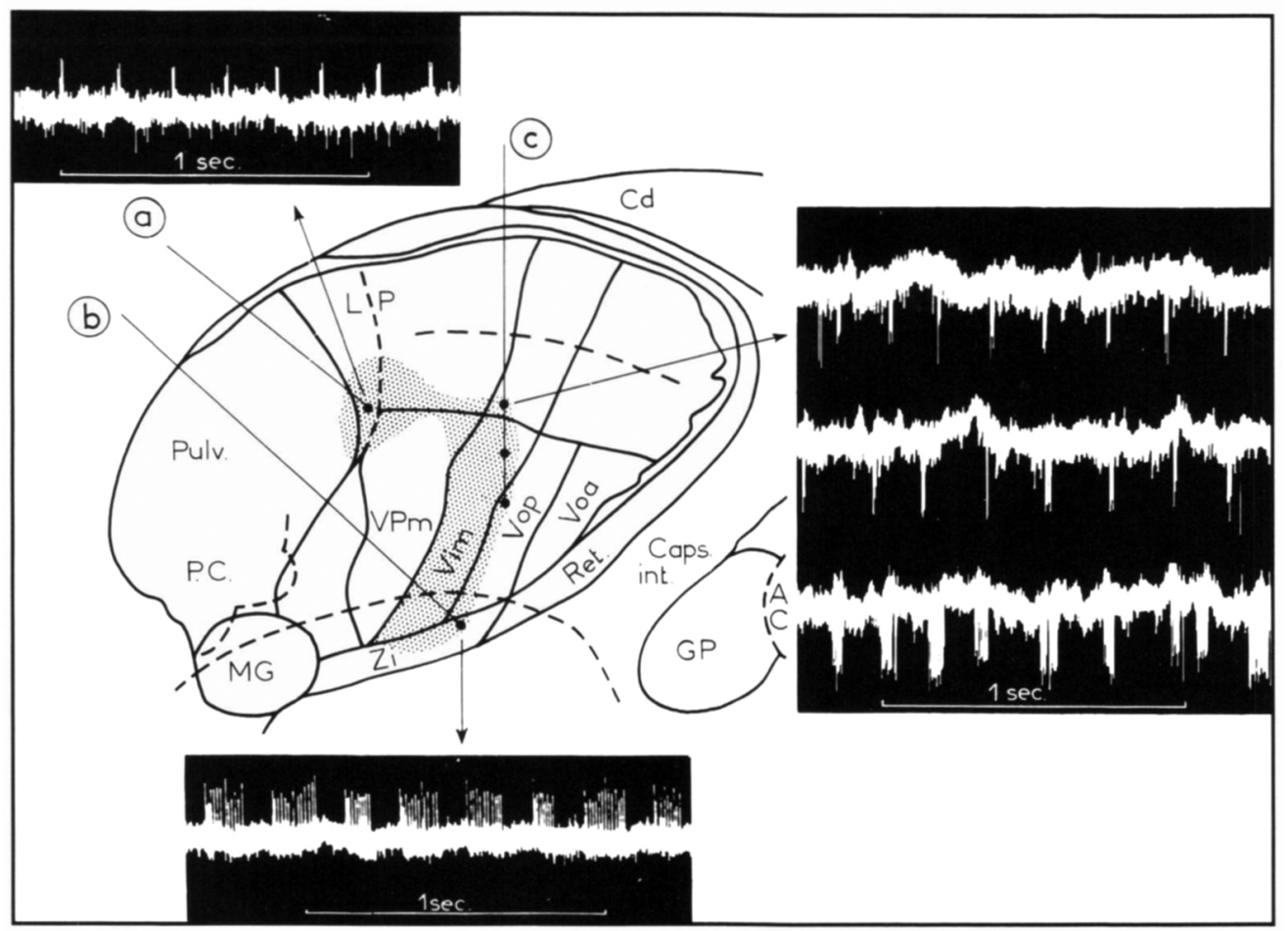

Figure 2 - Chez l'Homme autour du plan sagittal Latéral 15. (Atlas de Schaltenbrand and Bailey. 23) sont présentées des unités cellulaires ayant une activité rythmique dont la fréquence est voisine de celle du tremblement Parkinsonien. Les résultats regroupés ici proviennent de trois patients différents. chez lesquels la même région du Vim a été atteinte au moyen de trois trajectoires différentes de l'électrode $(a, b, c)$. Commissure antérieure $(A C)$ et postérieure $(P C)$. Noyaux thalaniques: Latéral postérieur $(L P)$, Pulvinar (Pulv.), Ventral postérieur, partie médiane (VPm), Ventral intermédiaire (Vim), ventral oral postérieur (Vop) é antérieur (Voa), Réticulé thalamique (Ret.). Genouillé médian (MG). Zona incerta (ZI), noyau Caudé (Cd), Globus pallidus (GP). Capsule interne (Caps int.). Toutes les cellules ayant un rythme de 4 à 5 par seconde et cette latéralité ont été observées par notre groupe dans la région en grisé (d'après 24).

l'avant à l'aire 3a. Les projections du thalamus vers l'aire 4 semblent maintenant un fait admis, même si les premières démonstrations de celui-ci sont souvent oubliées dans la littérature.

Pour expliquer l'activité des cellules du Vim pulsant à la fréquence du tremblement Parkinsonien, deux hypothèses pouvaient être avancées; ou bien il s'agissait de réponses provoquées par le tremblement, ou bien ou contraire les bouffées rythmiques étaient à l'origine du mouvement anormal. Pour au moins une partie de ces unités, la seconde explication nous a paru pouvoir être soutenue. En effet, au moment de l'exécution d'un mouvement volontaire, les bouffées observées pour une unité thalamique disparaissent juste avant celles du myogramme. Fréquemment aussi, aprés une période d'arrêt du tremblement, l'unité thalamique recommence à pulser avant que le tremblement n'apparaisse. Une étude systématique de ces activités rythmiques a été conduite par Jasper et Bertand,27 Bertrand et al, ${ }^{28}$ Ohye et al, ${ }^{29}$ Raeva. ${ }^{30}$ En utilisant des moyens informatiques, qui n'existaient pas lors de nos premières expériences, il a pu être ainsi démontré que les cellules rythmiques du Vim commandent bien les muscles et provoquent le tremblement, mais sont aussi sous le contrôle des afférences produites par le mouvement, faisant partie d'une longue boucle de rétroaction.

La physiologie animale devait permettre de résoudre définitivement ce problème. En effet lorsque nous commençions ces recherches sur l'homme, un groupe de chercheurs Canadiens avait déjà créé un modèle animal de la maladie de Parkinson. Louis Poirier, ${ }^{31}$ par des lésions placées au niveau du tronc cérébral, avait réussi à produire des tremblements ayant la fréquence du Parkinson chez le Macaque. Ces animaux enregistrés par Cordeau et al ${ }^{32}$ présentaient des activités rythmiques au niveau du cortex qui ressemblaient à celles que nous venions de trouver dans le Vim chez l'homme. Yves Lamarre poursuivit avec différents collaborateurs les enregistrements chez le singe trembleur. Ils montrèrent que les bouffées thalamiques ayant le rythme du tremblement parkinsonien existent chez le macaque tremblant, même lorsque celui-ci est curarisé. $33.34,35$ II en est de même lorsque l'animal a subi une section des racines dorsales. ${ }^{36}$ Les rythmes cellulaires enregistrés chez le malade Parkinsonien ne peuvent donc pas être dus aux messages renvoyés de la périphérie. Ainsi, l'expérimentation animale avait permis de répondre à une question posée par la recherche clinique: les rythmes centraux rencontrés dans le thalamus étaient dus à une anomalie de l'encéphale Parkinsonien. Mais quelle était l'origine de celle-ci?

Cette anomalie thalamique a pendant de longues années cessé d'occuper les chercheurs (voir cependant 29 et 30). La L-dopa thérapie était apparue et les opérations visant à soulager les Parkinsoniens avaient été en majorité abandonnées. Cependant, de nouvelles connaissances avaient été acquises et des faits anatomiques connus depuis longtemps avait repris de l'intérêt. Il était bien établi que les Parkinsoniens présentent une 


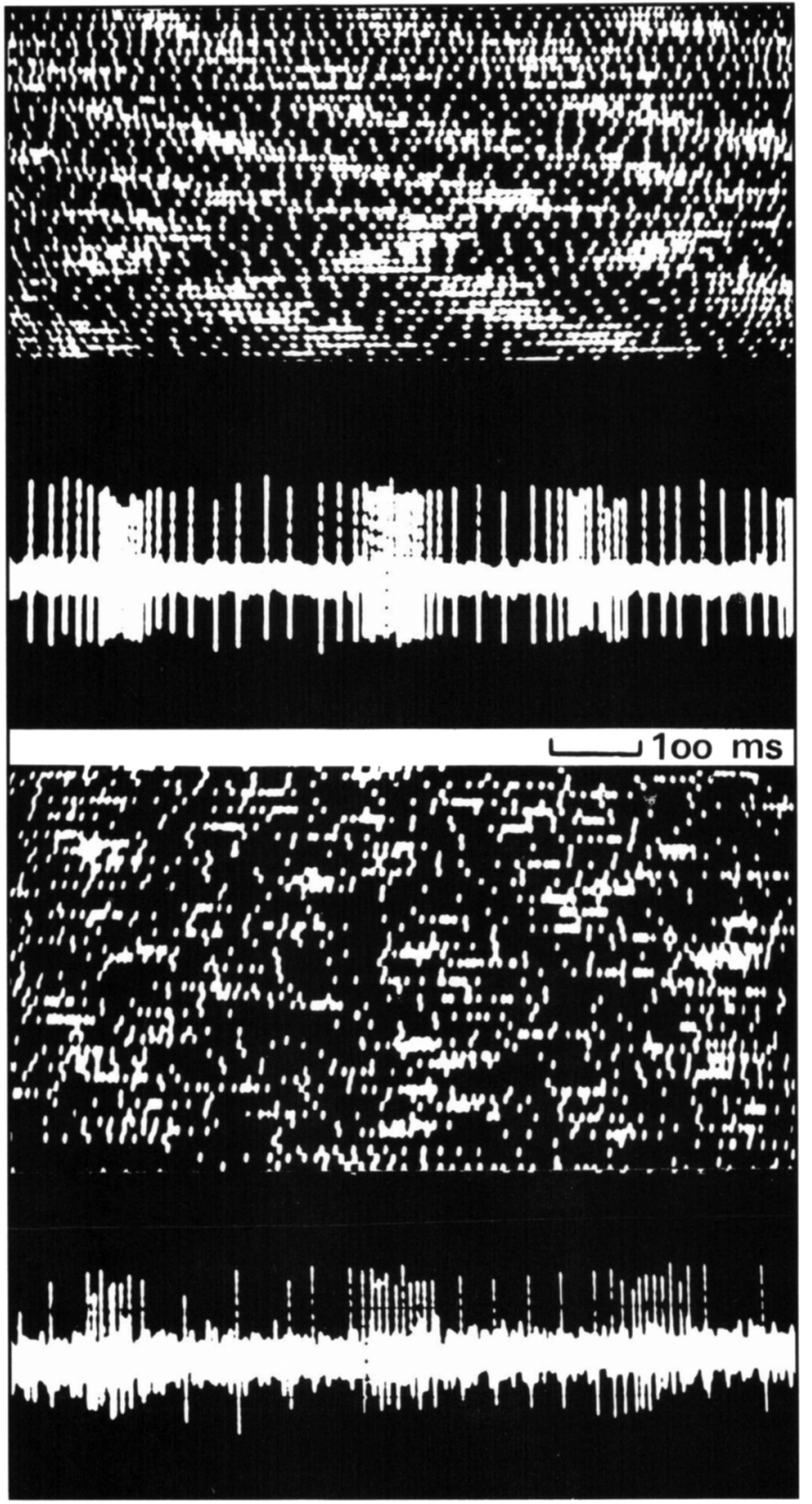

Figure 3 - Deux exemples de cellules, ayant un rythme voisin de celui du tremblement Parkinsonien, enregistrées dans la substance noire reticulata (Snr) chez des rats traités pendant 8 semaines par des injections journalières d'halopéridol. Dans les deux cas l'activité spontanée cellulaire est présentée sous forme de racé ainsi que de raster-display. Chez les animaux dont une voie striato-nigrale a été détruite par injection dans la substance noire compacse de 6 OHDopamine. l'acrivité de la majorité des cellules de la SNr est proche de celle présentée en has de la figure.

déplétion en dopamine des corps striés en même temps qu'une lésion des cellules de la substance noire compacte. L'existence d'une voie dopaminergique, reliant la substance noire aux corps striés, a été démontrée grâce aux méthodes de fluorescence. ${ }^{37,38}$ Par les méthodes électrophysiologiques Frigyesi et Purpura ${ }^{39}$ ainsi que notre groupe ${ }^{40}$ avaient confirmé son existence chez le Chat, puis chez le Rat. Les mêmes expériences mettaient également en évidence une voie descendante reliant les corps striés avec la substance noire. Celle-ci n'atteint pas, comme on a pu le croire un moment, les cellules de la substance noire compacte: elle se termine sur les cellules de la substance noire réticulée (SNr). A partir de cette structure les impulsions sont transmises au thalamus et au colliculus supérieur, puis de là, par des neurones branchés, vers le thalamus d'une part (le noyau ventral médian du rat, en particulier) et la moelle cervicale de l'autre. (voir références dans Deniau et al; ${ }^{41}$ Chevalier et Deniau). ${ }^{42}$

Dès lors, pouvait-on voir, dans un fonctionnement anormal de la substance noire réticulée après lésion de la substance noire compacte, l'origine des anomalies rencontrées chez l'Homme Parkinsonien ou les animaux trembleurs. Afin de nous en assurer, nous avons récemment étudié la $\mathrm{SNr}$ chez des rats ayant la voie nigrostriatale lésée par des injections localisées de 6hydroxy-dopamine, ainsi que chez des animaux ayant subi un long traitement par un neuroleptique, l'halopléridol. Dans les deux cas, les cellules de la SNr sont intactes, elles ont cependant une activité anormale, que l'absence ou la modification des contrôles qu'elles reçoivent à partir des corps striés peut expliquer. Il est intéressant de noter que les cellules de la $\mathrm{SNr}$, qui dans leur majorité ont chez l'animal normal une activité spontanée rapide et régulière, présentent chez les animaux ayant le système dopaminergique lésé des bouffées ayant un rythme de 3-5 par seconde 43 (figure 3). Nous avons d'autre part utilisé la dépression envahissante pour bloquer selectivement le striatum pendant un temps court et montré que l'activité des cellules de la SNr est seule modifiée pendant ce bloc, celle des cellules de la SNc restant inchangée. Une liaison se faisant au niveau de la $\mathrm{SN}$ entre les deux types cellulaires, souvent proposée sur la base de données biochimiques, ne semble donc pas mise en jeu dans ces conditions. Nous avons montré aussi que les contrôles exercés par le striatum sur la SNr sont complexes; ils font intervenir des inhibitions mais aussi des facilitations, et sont donc plus élaborés que l'inhibition produite par la voie gabaergique striato-nigrale. ${ }^{44}$ Il convient maintenant de poursuivre ces recherches chez le macaque, afin de comprendre quel mécanisme provoque le rythme anormal au niveau de la $\mathrm{SNr}$, et de confirmer que celui-ci est transmis au noyau Vim pour provoquer le tremblement.

\section{Recherches sur la douleur}

Celles-ci constituent un autre exemple d'interaction étroite entre Clinique et Recherche fondamentale. Il semble évident que des recherches sur la douleur ne peuvent être conduites sans que l'homme ne soit interrogé; c'est cependant à partir de données réunies chez l'animal que sont bâties la plupart des théories actuellement admises pour expliquer cette sensation. Les premières notions que nous avions réunies sur l'activation des cellules du thalamus médian, chez le chat puis chez le macaque, ont décidé de l'orientation ultérieure d'une partie des recherches de mon laboratoire. Avec divers collaborateurs nous avons étudié les caractéristiques des cellules de ces noyaux et des voies qui y conduisent.

- Ces structures reçoivent des messages apportés par des collatérales des fibres des cordons postérieurs (voir références dans 21). Celles-ci se projettent sur des cellules de la corne dorsale dont les fibres vont, en particulier chez le macaque, rejoindre le cordon antéro-latéral croisé. Ces messages sont donc conduits chez le primate par la voie spinothalamique, dont le rôle comme vecteur des messages douloureux a été depuis lors confirmé. 45 
- Les signaux qui atteignent ces structures sont conduits par des fibres $A \alpha \beta$ mais aussi par des fibres $A \delta$ et $C$ qui activent les cellules médullaires relayant les voies spinothalamiques. ${ }^{46}$

- Les stimulations naturelles qui activent les cellules du thalamus médian n'appartiennent pas au tact léger. Le plus souvent des tapes brèves, sont seules efficaces. Plus récemment l'arrivée à ce niveau d'afférences clairement nociceptives a été démontrée par d'autres groupes de chercheurs (voir références dans ${ }^{47}$ ).

- Avec L. Kruger, ${ }^{48}$ nous avons observé qu'au niveau des cellules du thalamus médian, deux réponses successives, séparées par un silence, sont provoquées par une stimulation unique. Le délai séparant les deux bouffées de réponses permettait de les attribuer l'une à des fibres du groupe A, l'autre à des fibres du groupe $C$. Elles rappelaient, par cette propriété, les caractéristiques de la double douleur que provoque chez l'homme une stimulation unique.

Un rôle spécial dans l'appréciation de la douleur a donc été proposé pour le thalamus médian. Cependant, ainsi que je l'ai fréquemment souligné, ce rôle ne peut être exclusif: en effet, la sensibilité douloureuse ressentie par l'homme normal est relativement bien localisée, alors que les champs récepteurs des cellules du thalamus médian ne présentent pas d'organisation topique. Il faut donc que le signe local soit apporté par l'activation d'autres structures. Nous verrons plus loin que celles alimentées par la voie néospinothalamique doivent sans doute jouer ce rôle.

Au cours de ces recherches, nous avons montré en outre que les réponses du thalamus médian peuvent s'observer, non seulement chez l'animal chloralosé, mais aussi chez l'animal implanté d'électrodes à demeure. Chez l'animal chronique, l'amplitude des activités évoquées varie considérablement avec l'état de vigilance de l'animal (figure 4). Faible lorsque celui-ci est attentif cette amplitude devient grande chez l'animal calme ou en sommeil avec onde lente pour disparaître à nouveau pendant le sommeil paradoxal. Ces fluctuations sont de sens opposé et plus importantes que celles observées dans les mêmes conditions, au niveau du thalamus primaire. Elles démontrent que des contrôles s'exercent sur les afférences qui atteignent le thalamus médian. Par des ablations localisées du cortex somato-moteur, il a été possible de faire disparaître ces fluctuations. ${ }^{49}$ Ces contrôles font donc intervenir des boucles corticales et nous avons insisté2l sur le rôle que ces boucles doivent jouer dans le filtrage des messages douloureux. Il est certain que ces effets d'origine corticale peuvent s'exercer au niveau de différentes structures, médullaires, mais aussi bulbaires et thalamiques. Les contrôles s'exerçant au niveau les plus centraux doivent intervenir dans les syndromes douloureux tout autant que les contrôles métamériques dont l'importance a surtout été jusqu'ici invoquée. ${ }^{50} \mathrm{Un}$ exemple en est donné par une observation clinique récente. 51 Lorsque la section d'un filet nerveux est accompagnée d'une douleur dans le territoire partiellement désafférenté, celle-ci peut être supprimée par toute stimulation capable de provoquer une sensation tactile dans le territoire qui avait perdu cette sensibilité. Les stimulations de l'extrêmité centrale du nerf sectionné, des racines dorsales et des cordons postérieurs ne sont efficaces pour supprimer la douleur que lorsque cette condition est remplie. Un contrôle inhibiteur, agissant au niveau médullaire peut être invoqué dans ces trois cas pour expliquer l'effet obtenu. Il n'en est pas de même quand l'analgésie est

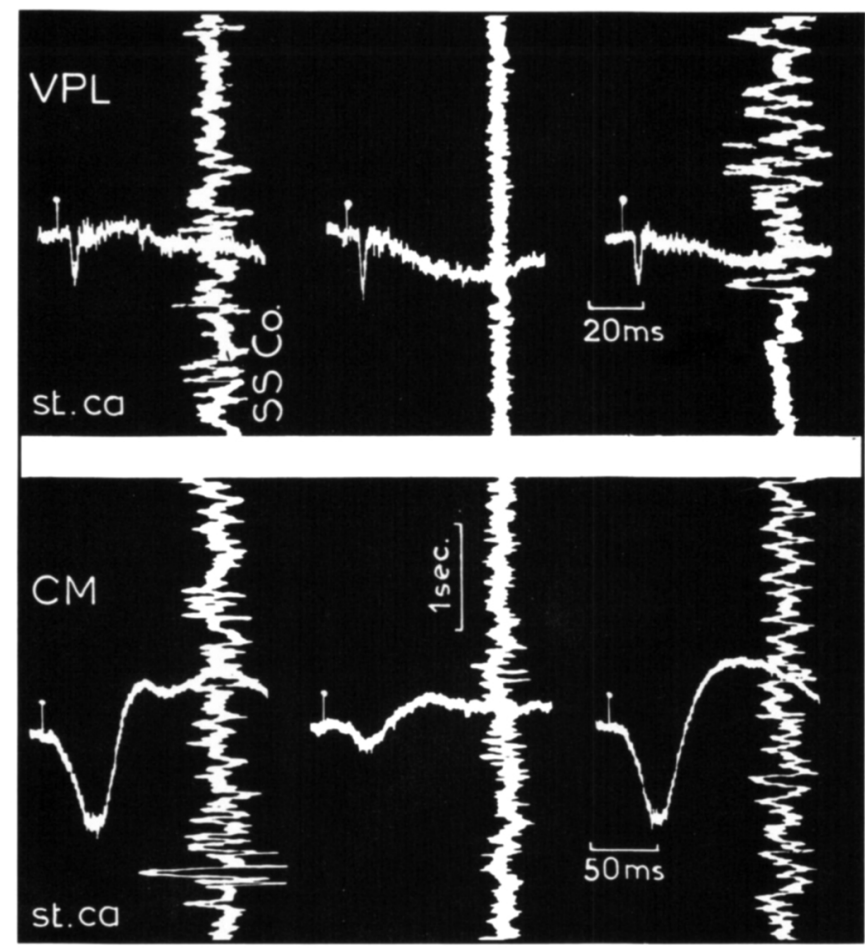

Figure 4 - Chez un chat implanté de deux électrodes bipolaires à demeure, dans le thalamus au niveau du noventral postérieur latéral (VPL) et du thalamus médian (CM). deux électrodes ont été aussi implantées sur le nerf radial intact afin de permetre sa stimulation électrique sans gêne pour l'animal. Les réponses de l'un et l'autre noyau ont été observées lors de stimulations de même intensité (tracés horizontaux). Deux électrodes corticales, implantées au niveau du gyrus suprasylvien (SSCo), permetraient de controler l'étar de vigilance de l'animal au cours de la période d'enregistrement (tracés verticaux). Lorsque ce tracé comportait des activités relative'ment lentes (6 à 8 par seconde) l'animal était calme et avait les yeux fermés, un tracé rapide correspondait par contre do un animal attentif à son environnement. Noter que les variations d'amplitude des réponses observées pendant ces deux stades comportementaux he sont importantes qu'au niveau du CM (d'après 21 ).

produite par la stimulation de la représentation thalamique primaire de la zone désafférentée au moyen d'électrodes implantées. Dès que l'intensité de la stimulation du n.VP provoque une sensation tactile le patient cesse de souffrir. II est impossible de faire appel, dans ce cas, à une inhibition purement métamérique et il convient de faire intervenir la boucle centrale dont nous venons de parler.

Les voies qui conduisent les afférences douloureuses vers le thalamus ont fait l'objet de nombreuses recherches anatomiques. Determinées par les méthodes de dégénérescence chez les primates, $52,53.54$ les terminaisons des voies conduites par le cordon antéro-latéral constituent deux entrées des afférences somatiques vers le thalamus, les contingents néospinothalamique et paléospinothalamique. Les projections néospinothalamiques viennent s'ajouter chez les primates à celles qui passent par les colonnes dorsales et atteignent le n.VP.; les paléospinothalamiques atteignent le thalamus médian. Il faut adjoindre à cette dernière projection les influx relayés dans la substance réticulée bulbomésencéphalique et dont l'électrophysiologie et l'anatomie ont montré qu'elles atteignaient aussi les cellules du thalamus médian. Quel rôle 
vont jouer ces deux systèmes de voies afférentes spinothalamiques dans l'appréciation des messages douloureux? Pour répondre à cette question, il était indispensable de connaitre les sensations provoquées chez l'homme par la stimulation de leurs structures centrales de projection. Je me référerai ici à une étude bibliographique récemment publiée sur ce sujet (voir dans ${ }^{47}$ ).

Il est connu depuis de longues années que l'on peut obtenir des sensations douloureuses par stimulation de l'encéphale. Toutefois ces stimulations ont été réalisées indifféremment chez des patients atteints d'affections douloureuses aussi bien que chez des patients atteints de maladies de la motricité, et une certaine confusion se dégage de l'ensemble des résultats. Les données deviennent au contraire plus claires lorsque les sensations obtenues chez les patients atteints d'un syndrome douloureux sont séparées de celles provoquées chez les patients qui ont pour la réception douloureuse un système nerveux intact. 45 Chez ces derniers, la douleur ne peut être produite que par des stimulations appliquées en des zônes localisées correspondant au passage des fibres spinothalamiques dans la moelle puis dans le bulbe et le mésencéphale, enfin en quelques points de la partie inféro-postérieure du n.VP. Il s'agit vraisemblablement dans tous ces cas de la mise en jeu de la projection néospinothalamique. Au niveau du cortex, d'après les données réunies par les neurologues et les neurochirurgiens qui ont eu l'occasion d'observer les auras douloureuses de certaines crises épileptiques, la région contenant les cellules qui recoivent ces messages douloureux se trouvent sur la lèvre postérieure de la scissure de Rolando (limite de l'aire 1 et de l'aire 3 ) où elles ont une organisation topique.

Les zones où se projettent les afférences paléospinothalamiques ont été aussi étudiées chez l'homme ayant un système nerveux intact. Des stimulations appliquées dans la partie médiane du tronc cérébral, en particulier dans la formation réticulée bulbo-mésencéphalique, provoquent surtout des sensations de brûlure et des troubles végétatifs; les régions thalamiques médianes ne donnent lieu qu'exceptionnellement à des sensations conscientes.

Les résultats sont totalement différents lorsque les stimulations sont appliquées chez des patients ayant subi une large désafférentation. Celle-ci peut être provoquée par des lésions apparaissant à des niveaux centraux aussi bien que périphériques. Nous nous sommes surtout intéréssés aux lésions plus centrales que le premier corps cellulaire. (après lésion de nerfs périphériques, en effet, des névromes peuvent se produire dont l'irritation vient troubler l'interprétation des résultats). Lorsqu'une désafférentation a privé une région périphérique de ses connexions centrales, la zone qui a perdu toute sensibilité provoquée, devient le siège de douleurs permanentes accompagnées de paroxysmes. La stimulation de l'encéphale chez ces patients, au niveau du thalamus aussi bien que du cortex, produit des sensations douloureuses. Celles-ci sont ressenties, le plus souvent, dans la zone périphérique qui a perdu ses afférences et la région centrale qui les provoque est plus large que celle qui, chez l'homme normal, représente la région périphérique désafférentée. Ces douleurs peuvent être obtenues à partir du thalamus primaire, de la capsule et du cortex somatomoteur mais aussi à partir de multiples points du thalamus médian, dans une région, qui lorsqu'elle est stimulée chez l'homme ayant un système de projection somatique intact ne provoque pratiquemment aucune sensation. Une transformation s'est donc produite, après la désafférentation, dans les caractéristiques du système nerveux central. Afin de mieux comprendre les problèmes que posent la douleur chez les patients désafférentés, et en particulier ceux qui soffrent d'un arrachement du plexus brachial, nous avons réalisé avec Blaine Nashold un modèle animal de ce syndrome. 55 L'arrachement chez l'homme de 4 à 5 racines dorsales, entre $\mathrm{C} 5$ et $\mathrm{TH} 1$, provoque des douleurs permanentes souvent insupportables, ressenties dans le creux de la main (région totalement désafférentée) auxquelles s'ajoutent des bouffées de douleurs paroxystiques de torsion ou de compression apparaissant dans tout le bras (région partiellement désafférentée). Les mêmes lésions de racines provoquent, lorsqu'elles sont pratiquées chez le rat, les mêmes désafférentations. Chez les animaux qui ont subi ces lésions apparaît, après quelques jours, un comportement d'automutilation qui conduit progressivement à l'ablation des doigts de la main puis de l'avant-bras. Nous avons étudié l'évolution de ces automutilations en chiffrant arbitrairement l'étendue de ces lésions. Ces automutilations ont été aussi étudiées chez les primates par d'autres auteurs. 56 Pour diverses raisons nous avons attribué ces automutilations au comportement d'un animal tentant de supprimer une zone dans laquelle il ressent une sensation insupportable, et non pas une région devenue insensible et inutile. En effet, dans les premiers jours qui suivent la section des racines, avant que ne se produise l'automutilation, l'animal continue à se servir du membre désafférenté. En outre, beaucoup de caractéristiques du développement de la douleur chez l'homme, rappellent celles du développement de l'automutilation chez l'animal. C'est ainsi, par exemple, que cette dernière évolue différemment au cours des mois de l'année et que des modifications similaires ont été récemment décrites pour les douleurs spontanées ressenties par l'homme. Enfin chez le rat des injections journalières de dolosal suppriment le comportement d'automutilation. Notre groupe a donc utilisé ces animaux comme modèles de douleur de désafférentation et nous avons étudié chez eux les modifications qui apparaissent dans les centres après la privation d'afférences. Contrairement à ce que l'on aurait pu attendre, celle-ci ne provoque pas le silence dans les aires centrales où les afférences se projettent normalement. Des activités, en bouffées plus ou moins régulières, apparaissent dans la moelle et le noyau cuneatus dès les premiers jours qui suivent la section des racines. Plus tard, des rythmes réguliers (environ 10 par seconde) se développent dans les zones désafférentées du thalamus primaire et dans le thalamus médian et le cortex somatomoteur. On comprend dès lors, que ces animaux aient des sensations, localisées par erreur, dans les régions périphériques ayant perdu leurs afférences. Des enregistrements pratiqués récemment par Tasker et ses collaborateurs ${ }^{57}$ ont montré que l'on trouve de telles activités organisées en bouffées anormales dans le thalamus des patients souffrant de douleurs de désafférentation. Le modèle animal que nous avons ainsi developpé à partir d'un modèle humain nous a déjà permis de rechercher le rôle que joue la boucle corticale de contrôle dans ce syndrome. ${ }^{58}$ Il est utilisé actuellement pour tester les drogues qui, supprimant le comportement d'automutilation, ont des chances d'abolir les douleurs de désafférentation chez les patients.

Aucun des ensembles de recherches que nous venons de résumer n'est terminé; il est donc prématuré d'affirmer qu'ils 
aboutiront à des méthodes permettant d'améliorer l'état des patients. Nous avons vu cependant combien la stéreotaxie chez l'Homme a bénéficié des études qui avaient été conduites pour comprendre l'organisation thalamique chez l'animal. Nous avons vu aussi comment, pour tenter d'expliquer les anomalies rencontrées dans l'encéphale humain, deux champs nouveaux ont été ouverts à la recherche fondamentale. Soulignons, en terminant, que nous n'avons traité ici que de deux exemples et que bien d'autres problèmes se posent, qui ne pourront être résolus que par la collaboration étroite entre cliniciens et fondamentalistes.

\section{RÉFÉRENCES}

1. Brock LG, Coombs JS, Eccles JC. The recording of potentials from motoneurones with an intracellular electrode. J Physiol, London 1952; 117: 431-460.

2. Albe-Fessard D, Buser P. Explorations de certaines activités du cortex moteur chez le chat par microélectrodes. J Physiol, Paris 1953; 46: 14-16.

3. Mountcastle VB, Henneman E. Pattern of tactile representation in thalamus of cat. J Neurol 1949; 12:85-100.

4. Mountcastle VB, Henneman E. The representation of tactile sensibility in the thalamus of the monkey. J of Comp Neurol 1952; 97: 409-440.

5. Rose JE, Mountcastle VB. Activity of single neurons in the tactile thalamic region of the cat in response to a transient peripheral stimulus. Bull. Johns Hopkins Hospital 1954; 94: 238-282.

6. Adrian ED. Afferent discharges to the sensory cortex from peripheral sense organ. J Physiol, London 1941; 100: 159-190.

7. Marshall WH, Woolsey CN, Bard P. Observations on cortical somatic sensory mechanisms of cat and monkey. J Neurophysiol 1941; 4: 1-25.

8. Woolsey CN, Fairman D. Contralateral, ipsilateral and bilateral representation of cutaneous receptors in somatic areas I and II of the cerebral cortex of pig, sheep and other mammals. Surgery, St Louis 1946; 19: 684-702.

9. Amassian VE. Studies on organization of a somesthesic association area, including a single unit analysis. J Neurophysiol 1951; 14: 433-444.

10. Jasper HH, Ajmone-Marsan C. A stereotaxic atlas of diencephalon of the cat. The national research council of Canada, Ottawa 1954; pp 71 .

11. Albe-Fessard D, Rougeul A. Activités d'origine somesthésiques evoquées sur le cortex non-spécifique du chat anesthésié au chloralose. Rôle du centre Médian du thalamus. Electroencephalog and Clin Neurophysiol 1958; 10: 131-152.

12. Albe-Fessard D, Besson JM. Convergent thalamic and cortical projections - The non-specific system. In: Iggo A, ed. Handbook of Sensory Physiology, vol. II, Somatosensory System. SpringerVerlag, Berlin, Heidelberg, New York 1973: 490-560.

13. Magoun HW, Mac Kinley WA. The termination of ascending trigeminal and spinal tracts in the thalamus of the cat. Amer J Physiol 1942; 137: 409-416.

14. Albe-Fessard D, Arfel G, Guiot G, Hardy J, Vourc'h G, Hertzog E, Aléonard P. Dérivations d'activités spontanées et évoquées dans les structures cérébrales profondes de l'homme. Revue Neurol 1962; 106: 89-105

15. Albe-Fessard D, Argel G, Guiot G. Activités électriques caractéristiques de quelques structures cérébrales chez l'homme. Ann Chirur 1963; 17: 1185-1214.

16. Horsley W, Clarke RH. The structure and function of the cerebellum examined by a new method. Brain 1908; 31: 54-124.

17. Percheron G, Lacourly N, Albe-Fessard D. Lack of precision of thalamic stereotaxy based on cranial landmarks in some species of Macaca. Medical primatology; Proc 3rd conf med surg. Primates, Lyon Part II, Karger, Basel 1972: 297-304.
18. Percheron G. Ventricular landmarks for thalamic stereotaxy in Macaca. J Med Primatol 1975; 4: 217-244.

19. Talairach J, de Ajuriaguerra J, David M. Etude stéréotaxique des structures encéphaliques profondes chez l'Homme. Presse médicale 1952; 60: 605-609.

20. Féger J, Ohye C, Gallouin F, Albe-Fessard D. Stereotaxic technique for stimulation and recording in non-anesthetised monkeys: application to the determination of connections between caudate nucleus and substantia Nigra. Advances in Neurology. vol. 10, Meldrum BS and Mardsen CD, eds. Raven Press, New York 1975: 35-45.

21. Albe-Fessard D. Organization of somatic central projections. In: Neff, ed. Contributions to Sensory Physiology. New York, Acad. Press, vol. Il 1967: 131-167.

22. Albe-Fessard D, Guiot G, Lamarre Y, Arfel G. Activation of thalamocortical projections related to tremorogenic processus. $/ \mathrm{n}$ : Purpura and Yahr, eds. The Thalamus, Columbia University Press 1966: 237-253.

23. Schaltenbrand G, Bailey P. Introduction to stereotaxis with an Atlas of the Human Brain. Grune and Stratton: New York 1959: $494 \mathrm{pp}$.

24. Albe-Fessard D. Physio-Pathologie du Parkinson. In: Le point sur la maladie de Parkinson Merck Sharp et Dohme, eds. Bruxelles 1973: 1-20.

25. Woolsey CN, Chang HT, Bard P. Distribution of cortical potentials evoked by electrical stimulation of dorsal roots in Macaca Mulatta. Fed Proc 1947; 6: 230.

26. Albe-Fessard D, Liebskind J. Origine des messages somatosensitifs activant les cellules du cortex moteur chez le Singe. Exp Brain Res 1966; 1: 127-146.

27. Jasper $\mathrm{HH}$, Bertrand $\mathrm{C}$. Thalamic units involved in somatic sensation and voluntary and involuntary movements in man. $I n$ : Purpura and Yahr MD, eds. The Thalamus, Columbia University Press, New York 1966: 365-390.

28. Bertrand G, Jasper H, Wong A, Mathews G. Microelectrode recording during stereotaxic surgery. Clin Neurosur 16: 328-355.

29. Ohye C, Fukumashi A, Narabayashi H. Spontaneous and evoked activity of sensory neurons and their organization in the human thalamus. Z Neurol 1972; 203: 219-234.

30. Raeva $S$. Localization in human thalamus of units triggered during verbal commands, voluntary movements and tremor. Electroencephal and Clin Neurophysiol 1986; 63: 160-173.

31. Poirier LJ. Experimental and morphological studies of midbrain dyskinesias. J Neurophysiol 1960; 23: 534-545.

32. Cordeau JP, Gybels J, Jasper H. Microelectrode studies of unit discharges in the sensory motor cortex. Investigation in monkeys with experimental tremor. Neurology 1960; 10: 591-600.

33. Lamarre $Y$, Dumont $M$, Joffroy AJ. Le tremblement de type parkinsonien et l'action de l'harmaline. Faits expérimentaux et mécanismes hypothétiques. Arch Ital Biol 1973; 111: 493-503.

34. Lamarre $\mathrm{Y}$, Joffroy AJ. Rhythmic bursting unit potentials in the ventrolateral thalamus of the chronic monkey. Int J Neurol 1971; 8: 190-197.

35. Joffroy AJ, Lamarre $Y$. Rhythmic unit firing in the precentral cortex in relation with postural tremor in a deafferented limb. Brain Res 1971; 27: 386-389.

36. Ohye C, Bouchard R, Larochelle L, Bédard R, Boucher R, Raphy B, Poirier LJ. Effect of dorsal rhizotomy on postural tremor in monkey. Exp Brain Res 1970; 10: 140-150.

37. Dahlstrom A, Fuxe K. Evidence for the existence of monoamine containing neurons in the central nervous system; I . Demonstration of monoamines in the cell bodies of brain system neurons. Acta Physiol Scand, 1964, 62, (Suppl: 62) 232: 1-55.

38. Anden NN, Carlsson A, Dahlstrom A, Fuxe K, Hillarp NA, Larsson $\mathrm{K}$. Demonstration and mapping out of nigroneostriatal dopamine neurons. Life Sci 1964; 3: 523-550.

39. Frigyesi TL, Purpura DP. Electrophysiological analysis of reciprocal caudato-nigral relations. Brain Res 1967; 6: 440-456. 
40. Albe-Fessard D, Raeva S, Santiago W. Sur les relations entre la Substance Noire et le Noyau Caudé. J Physiol, Paris 1967; 59 : 324-325.

41. Deniau JM, Chevalier G, Féger J. Electrophysiological study of the nigrotectal pathway in the rat. Neuroscience Letters 1978; 10: 215-220.

42. Chevalier G, Deniau JM. Spatiotemporal organization of a branched tecto-spinal/tecto-diencephalic neuronal system. Neuroscience 1984; 12: 427-439.

43. Sanderson $P$, Mavoungou R, Albe-Fessard D. Changes in substantia Nigra pars reticulata activity following lesions of the substantia nigra pars compacta. Neuroscience Letters 1986; 67: 25-30.

44. Albe-Fessard D, Sanderson P. A demonstration of tonic inhibitory and facilitatory striatal actions on substantia Nigra neurons. In: Carpenter M and Jaramian A, eds. The Basal Ganglia II, Plenum Publishing Corporation 1987: 321-326.

45. Tasker RR, Organ LW, Hawrylyshyn PA. The thalamus and midbrain of Man. C. Thomas Pub. Springfield, Illinois, $505 \mathrm{pp}$.

46. Albe-Fessard D, Levante A, Lamour Y. Origin of spinothalamic tract in monkeys. Brain Research 1974; 65: 503-509.

47. Albe-Fessard D, Berkley KJ, Kruger HJ, Ralston III HJ, Willis W. Diencephalic mechanism of pain sensation. Brain Research Reviews 1985; 9: 217-296.

48. Albe-Fessard D, Kruger L. Duality of unit discharges from cat centrum medianum to natural and electrical stimulation. J Neurophysiol 1962; 25: 3-20.

49. Guilbaud G, Menetrey D. Rôle joué par les voies et aires de projection lemniscales dans le contrôle des afférences extralemniscales au cours du sommeil naturel chez le chat. Electroenceph and Clin Neurophysiol 1970; 29: 295-302.

50. Melzack R, Wall PD. Pain mechanisms: a new theory. Science 1965; 150: 971-979.

51. Albe-Fessard D, Tasker R, Yamashiro Y, Dostrovsky J. Effects of stimulation of the primary thalamic somatic relay for the relief of central pain. The Pain Clinic II. Ph Scherpereel et al, eds, VNO Science Press, Utrecht 1987: 207-213.

52. Mehler WR. Some neurological species differences - a posteriori. Ann NY Acad Sci 1969; 167: 424-468.

53. Bowsher $\mathrm{D}$. Termination of the central pain pathways in man: the conscious appreciation of pain. Brain 1957; 80: 606-622.

54. Boivie $\mathbf{J}$. An anatomical reinvestigation of the termination of the spinothalamic tract in the monkey. J Comp Neurol 1979; 186: 343-370.

55. Lombard MC, Nashold BS, Albe-Fessard D. Deafferentation hypersensitivity in the rat after dorsal rhisotomy: a possible animal model of chronic Pain. Pain 1979; 6: 163-174.

56. Levitt M. Dysesthesias and self-mutilation in humans and subhumans. A review of clinical and experimental studies. Brain Research Rev 1985; 10: 247-290.

57. Gorecki JP, Tasker RR, Hirayama T, Dostrovski J. Microelectrode recording from the deafferented thalamus. In: Narabayashi, ed. Workshop on Neurophysiological Recordings During Stereotaxic Neurosurgery. Applied Neurophysiol 1988; souspresse.

58. Rampin O, Morain P. Cortical involvement in dorsal horn cell hyperactivity and abnormal behavior in rats with dorsal root section. Somatosensory Research 1987; 4: 237-251. 